\title{
Constraining the emission of cosmic electrons from supernova remnants with flux, dipole anisotropy and radio data
}

\author{
Silvia Manconi ${ }^{* \dagger}$ \\ University of Torino and INFN, Torino \\ E-mail: manconi@to.infn.it
}

\begin{abstract}
In this contribution an interpretation of the most recent data on cosmic-ray electron and positron (CRE) fluxes (from Fermi-LAT, HESS, CALET and AMS-02), with a special focus on the electron contribution from supernova remnants (SNRs), is presented. For the first time, the constraints coming from CRE flux up to $20 \mathrm{TeV}$, as well as Fermi-LAT dipole upper limits and radio measurements of individual nearby SNRs are consider together. It is discussed how CRE data up to $20 \mathrm{TeV}$ can constrain the energy cutoff of the electron emission from a smooth distribution of SNRs in the Galaxy. Also, the consequences of the recent Fermi-LAT measurement of the dipole anisotropy are explored, studying in particular the total emission energy in electrons and spectral index of Vela YZ and the Cygnus Loop SNRs. Finally, the full radio spectrum of nearby SNRs is used to constrain their electron injection spectrum. Results show how the combination of constraints from different observables shed light on the interpretation of present CRE data.
\end{abstract}

7th Fermi Symposium 2017

15-20 October 2017

Garmisch-Partenkirchen, Germany

${ }^{*}$ Speaker. 


\section{Introduction}

Data on cosmic ray electron and positron fluxes (CRE) are an incredible window to explore the local (few kpc) Galactic enviroment. In fact, as opposed to protons, the energy losses timescale for CRE is smaller than the diffusion one. Recently, high precision CRE data from AMS-02 [1], FermiLAT [2], and preliminary data from HESS [3] and CALET[4] experiments have been presented up to few tens of $\mathrm{TeV}$, as well as upper limits for the dipole anisotropies [5]. In this work the CRE flux data, as well as dipole upper limits and radio measurements of nearby individual sources, are consider together in order to constrain the contribution of SNRs.

\section{A model for cosmic ray electrons sources and propagation}

The model used for the sources and for the propagation of CRE is based on [6], to which we address for any detail (see also [7]). Supernova Remnants (SNRs) are considered the best candidate to accelerate Galactic CR thought diffuse shock acceleration, and are included in our model as sources of $e^{-}$. In addition, Pulsar Wind Nebulae (PWNe) produce either $e^{+}$and $e^{-}$in the strong magnetic field that surrounds the pulsars that are then accelerated up to high energies with relativistic shocks. The source of the secondary component for both $e^{+}$and $e^{-}$is the spallation of hadronic $\mathrm{CR}$ species in the interstellar medium. Once they are produced, $e^{+}$and $e^{-}$propagate in the Galaxy and are affected by different process, in particular by the radiative losses in the interstellar radiation fields and the diffusion in the irregularities of the interstellar magnetic field. Above energies larger than few $\mathrm{GeV}$, the propagation of CRE in the Galaxy is described by means of the transport equation for $\psi=\psi(E, \mathbf{x}, t) \equiv d n / d E$ :

$$
\frac{\partial \psi}{\partial t}-\nabla \cdot\{K(E) \nabla \psi\}+\frac{\partial}{\partial E}\left\{\frac{d E}{d t} \psi\right\}=Q(E, \mathbf{x}, t)
$$

where the term $Q(E, \mathbf{x}, t)$ includes all the possible sources. The diffusion is described in Eq.2.1 by means of the diffusion coefficient $K(E)=K_{0} E^{\delta}$, while the energy losses due primarily to inverse Compton scattering and synchrotron emission are included in $d E / d t$. To solve Eq.2.1 we use a semi analytical model, where the diffusive halo is modeled as a thick disk of radius $20 \mathrm{kpc}$ and vertical half height $L \sim 1-15 \mathrm{kpc}$. As for the propagation parameters $\left(\delta, K_{0}, L\right)$, the results of the recent analysis of B/C AMS-02 data in [8] (Kappl+15) and [9] (Genolini+15) are used.

As described in [6], a smooth distribution of SNRs following a radial profile taken from [10] (G15) is considered, as well as single SNRs taken from the Green catalog [11]. When the single SNRs with distance $d<R_{\text {cut }}$ are inserted in the model, the smooth distribution is cutted to become the far SNRs contribution for $R=\left|r-r_{\odot}\right|<R_{\text {cut }}$. The injection spectrum for both SNRs and PWNe is taken as $Q(E)=Q_{0}\left(\frac{E}{E_{0}=1 \mathrm{GeV}}\right)^{-\gamma} \exp \left(-\frac{E}{E_{c}}\right)$, where the normalization $Q_{0}$ is constrained by using catalog quantities for single SNRs and PWNe, or using average characteristics for the smooth SNRs component. The contribution of PWNe is computed selecting single pulsars from the ATNF catalog with ages $T>50 \mathrm{kyr}$. A common efficiency of conversion $\eta$ of the spin down energy and spectral index $\gamma_{P W N e}$ are fitted to data. The computation of the secondary component closely follows the method described in [12], where the primary CR fluxes are taken from a fit to AMS-02 data.

An additional observable that we use to test our models is the dipole anisotropy of the CRE fluxes (see [6] and references therein). In fact, when a single or few single sources are introduced to explain an important part of the CRE fluxes, a dipole anisotropy may arise in the observed fluxes. 
To compute the dipole anisotropy from a single source at distance $d$ that produces a number density $\psi_{e^{+}+e^{-}}(E)$ we use the equation:

$$
\Delta(E)_{e^{+}+e^{-}}=\frac{3 K(E)}{c} \frac{2 d}{\lambda^{2}\left(E, E_{S}\right)} \frac{\psi_{e^{+}+e^{-}}(E)}{\psi_{e^{+}+e^{-}}^{t o t}(E)},
$$

that is valid in the diffusion propagation regime. The $\lambda\left(E, E_{S}\right)$ is the typical propagation scale (see Eq. 2.11 of [6]) and $\psi_{e^{+}+e^{-}}^{\text {tot }}(E)$ refers to the total $e^{+}+e^{-}$number density, including both the contributions from isotropic smooth populations as well as single sources. As discussed in [6], we compute anisotropy predictions only for the models compatible with CRE data, and we then compare our results to the anisotropy upper limits.

\section{The injection energy cutoff of SNRs}

To explore how new CRE data up to $20 \mathrm{TeV}$ can constrain the energy cutoff of the electron emission from a smooth distribution of SNRs in the Galaxy, a simple model is firstly considered. The aim of this analysis is to find a lower limit for the energy cutoff of the SNRs contribution. The SNRs distribution is thus modeled without any radial cut, and the PWNe emission together with the secondary production of $e^{+}$and $e^{-}$are also included. The spatial distribution of SNRs is taken with a given energy cutoff $E_{c}$ and following the G15 radial profile, while the spectral index and the normalization are fitted to the data. Additional free parameters are the spectral index and normalization of the PWNe, and a re-normalization factor for the secondary component. This model is fitted to the complementary data set of the AMS-02 data for $e^{+}+e^{-}$and $e^{+}$and the preliminary HESS data for $e^{+}+e^{-}$for $E>10 \mathrm{GeV}$. Note that the systematic band in the HESS preliminary data is an effect primarily on the energy scale, see [13]. Different values for the $E_{c}$ of the SNRs contribution from $5 \mathrm{TeV}$ up to $50 \mathrm{TeV}$ are used, and both the propagation models Genolini+15 and Kappl+15 have been considered. Regardless the propagation parameters, the result is that only models with an energy cutoff $E_{c}>10 \mathrm{TeV}$ for the SNRs component give a flux of $e^{+}$and $e^{-}$that is compatible with the considered data set.

\section{Unconstrained local SNRs}

For energies higher than few tens of $\mathrm{GeV}$ the hypothesis of a smooth, uniform distribution of CRE sources in the local Galaxy is strongly limited (see e.g. $[14,6]$ and references therein). The smooth distribution of SNRs is thus considered here with a radial cut of $R_{\text {cut }}=0.7 \mathrm{kpc}$, becoming the far SNRs component. A near component is included by means of the contribution of the Vela YZ and Cygnus Loop SNRs, that given their age and distance could emerge as dominant sources in the high energy range (see [6] for more details and for the used distances and ages). In this analysis the emission of $e^{-}$from these local SNRs is studied with only CRE data, including the upper limits on dipole anisotropy. The spectral index and normalization of Vela YZ and Cygnus Loop are thus fitted to the data, together with the free parameters already considered for the analysis in Sec.3. In particular, a wide range for the spectral index for Vela YZ of [1.90,3.10] and Cygnus of $[1.5,2.5]$ are considered. The results of the fit to the combined data set of AMS-02 and HESS preliminary data are shown in Fig. 1 for $e^{+} e^{-}$(left panel) and $e^{+}$(right panel) for the Kappl+15 propagation model. A $\chi_{\text {red }}^{2}<1$ is found for both Kappl+15 and Genolini+15 propagation models. The best fit to the data is shown as the black solid line, while the cyan band is the $2 \sigma$ interval. As a comparison, the data on $e^{+}+e^{-}$from the Fermi-LAT experiment [2] as well as the preliminary 
data from the CALET [4] are also plotted. For the $e^{+}$, as shown by Fig.1 (right panel) the PWNe component (blue dash dotted line) is dominant starting from $E>50-60 \mathrm{GeV}$. A best fit index of $\gamma_{P W N e}=1.79 \pm 0.03$ and an efficiency conversion of $\eta=0.056 \pm 0.004$ are found for the sum of catalog PWNe. As shown by Fig.1 (left panel) the unconstrained local SNRs emerge as a dominant component of the $e^{+}+e^{-}$flux starting from $E>40 \mathrm{GeV}$. In particular, the Vela YZ (black dotted line) is dominating the flux up to few $\mathrm{TeV}$, while the Cygnus Loop (magenta dash dotted line) contribution is dominant in the multi $\mathrm{TeV}$ range for the best fit case, even if between $2 \sigma$ (cyan band) can contribute up to one order of magnitude less, given the HESS uncertainty band. As for the injection spectrum parameters, the best fit spectral index for both the local sources tend to the borders of the allowed range, while the normalization is $Q_{0} \sim 10^{52} \mathrm{GeV}^{-1}$ for Vela YZ and $Q_{0} \sim 10^{47} \mathrm{GeV}^{-1}$ for Cygnus. The resulting dipole anisotropies for the single sources in this model
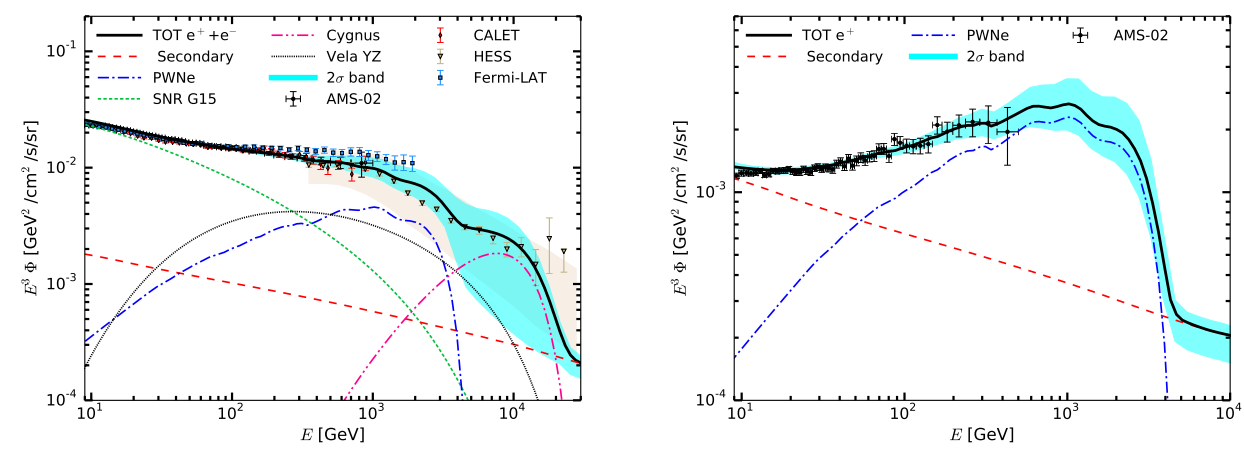

Figure 1: Results for the fit to the complementary AMS-02 data and HESS preliminary data for $e^{+}+e^{-}$ (left panel) and AMS-02 for $e^{+}$(right panel) fluxes using the model described in Sec. 4.

are shown in Fig.2 (left panel) as a function of the energy $E$ together with the upper limits coming from Fermi-LAT [5]. The black solid line represents the dipole anisotropy of Vela YZ for the best fit, while the dot dashed magenta line is for the best fit contribution of Cygnus Loop. The best fit to CRE data for the model with the unconstrained Vela YZ is excluded by the Fermi-LAT upper limits up to $800 \mathrm{GeV}$. In particular, the predicted anisotropy at $100 \mathrm{GeV}$ is $\Delta_{e^{+}+e^{-}} \sim 3 \cdot 10^{-2}$, thus a factor of five higher with respect to the upper limits. A number of models that are compatible with the flux data are thus exluded by the anisotropy analysis, showing how the emission from local SNRs is constrained with only data on CRE. Forthcoming analysis will include a study of the parameter space for the Vela YZ that is excluded by the dipole upper limits. When considering the resulting dipole anisotropy from the collection of all single sources in the model (blue dashed line in Fig.2, left panel), the interplay between Vela and Cygnus give $\Delta_{e^{+}+e^{-}} \sim 6 \cdot 10^{-2}$ at $10 \mathrm{TeV}$, a range that will be explored by the CALET experiment [4].

\section{Constraints from radio measurements}

In this section radio measurements of Vela YZ and Cygnus are considered to constrain their $e^{-}$emission. For more details on the connection between the radio spectrum of near SNRs and the injection spectrum of $\mathrm{CR} e^{-}$emission see [7]. For each source, the integrated flux density $B_{r}$ as a function of the frequency $v$ is fitted by means of Eq.2.9 in [6] to find best fit values and $3 \sigma$ allowed regions for the spectral index of the $e^{-}$population $\gamma_{S N R}$ and the normalization fo the injection 

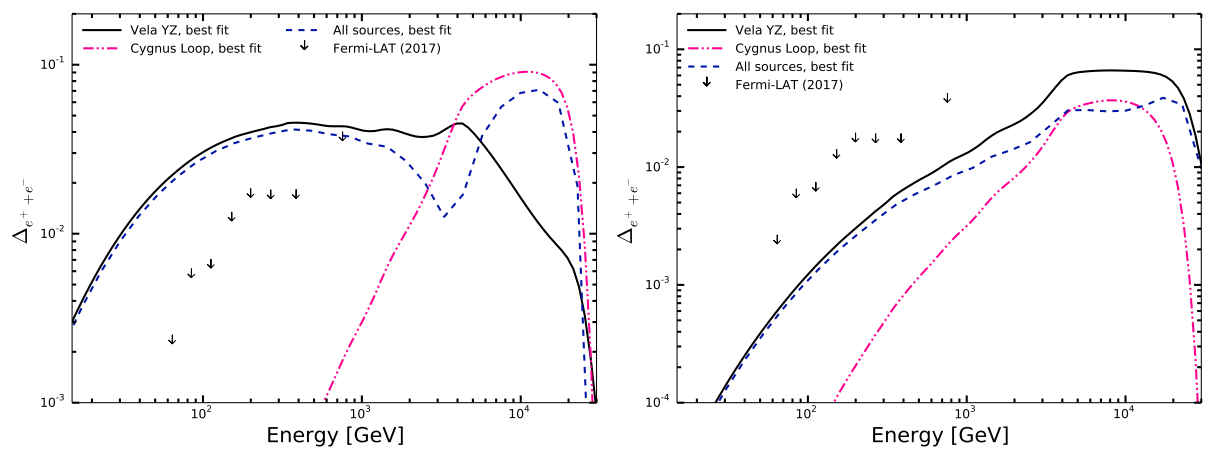

Figure 2: Predictions for the dipole anisotropies from Vela $\mathrm{YZ}$ and Cygnus Loop for the models described in Sec.4 (left) and Sec.5 (right panel) together with the upper limits from [5], Kappl+15 propagation model.

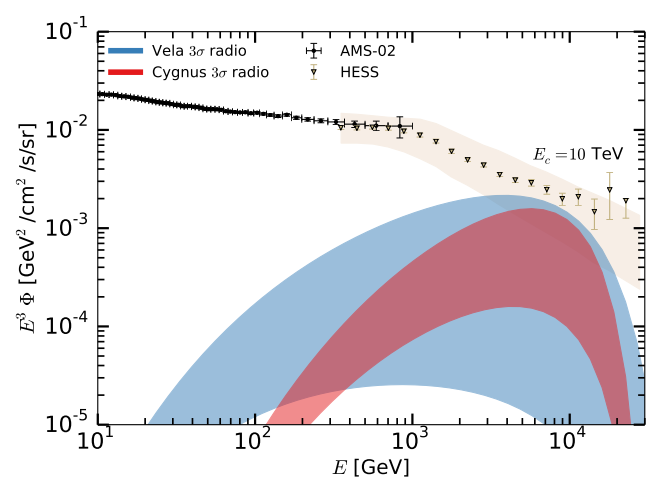

Figure 3: Expected CR $e^{-}$flux from Vela YZ (blue band) and Cygnus Loop (red band) if radio constraints for the spectral index and the normalization of the source injection spectrum are considered. Fluxes are computed for the Kappl+15 propagation model.

spectrum $Q_{0}$. The radio spectrum is taken from [15] for Vela YZ, and from [16] for Cygnus Loop. The value of the magnetic field is fixed to $60 \mu \mathrm{G}$ for Cygnus (see gamma ray studies in [17]) and $36 \mu \mathrm{G}$ for Vela YZ [18]. The results for the $3 \sigma$ regions for $\gamma_{\mathrm{SNR}}$ and $Q_{0}$ obtained from the radio spectrum are used to compute the corresponding $e^{-}$fluxes in Fig.3. The relative contribution to the $e^{+}$and $e^{-}$data is now constrained to be significantly lower with respect to what is found in Sec.4. For example, at $E \sim 800 \mathrm{Gev}$ the flux from Vela (Cygnus) is now constrained to be between the 30\% (4\%) and less than the $1 \%$ of the data. The obtained constraints for $\gamma$ and $Q_{0}$ for both sources are included in the model to fit again the combined AMS-02 and HESS data sets. Apart for the inclusion of radio constraints for the local sources, the model for CRE is analogous to what described in Sec.4. A best fit is found with $\chi_{\text {red }}^{2}<1$, and the corresponding dipole anisotropies for Vela YZ and Cygnus are reported in Fig.2 (right panel). Taking into account the constraints from radio measurements, the resulting model for near SNRs is automatically consistent with FermiLAT upper limits. In fact, the anisotropy from Vela YZ is now constrained to be at least a factor of $\sim 2$ lower with respect to the upper limits up to $1 \mathrm{TeV}$.

\section{Questions \& Answers}

1)Question/comment: Since the preliminary spectrum measured by HESS is without spectral features, you are obtaining best fit models without spectral features. A:Since we are fitting the data 
including the systematic band, conclusions regarding possible spectral features in the $\mathrm{TeV}$ range are not yet precise. Future data in the $\mathrm{TeV}$ range from experiments as DAMPE, ISS-CREAM, CALET will shade light on possible spectral features with more precision. 2)Q: Is the radio spectrum of SNRs a good description of the released electrons? A:We work under the assumption that the characteristics of the population of $e^{-}$emitting in the radio band are similar to the $e^{-}$released in a burst-like event in the interstellar medium. In reality, the release is a time and energy dependent process. Nevertheless, providing that the release time is small compared to the age of the source, this is a reasonable approximation.

\section{References}

[1] M. Aguilar et al., Precision Measurement of the $\left(e^{+}+e^{-}\right)$Flux in Primary Cosmic Rays from $0.5 \mathrm{GeV}$ to 1 TeV with AMS-02, Physical Review Letters 113 (Nov., 2014) 221102.

[2] S. Abdollahi, M. Ackermann, M. Ajello et al., Cosmic-ray electron-positron spectrum from $7 \mathrm{GeV}$ to 2 TeV with the Fermi Large Area Telescope, Phy. Rev. D 95 (Apr., 2017) 082007.

[3] https://indico.snu.ac.kr/indico/event/15/session/5/contribution/694.

[4] https://indico.snu.ac.kr/indico/event/15/session/23/contribution/416.

[5] S. Abdollahi et al., Search for Cosmic-Ray Electron and Positron Anisotropies with Seven Years of Fermi Large Area Telescope Data, Phys. Rev. Lett. 118 (2017) 091103, [1703. 01073].

[6] S. Manconi, M. Di Mauro and F. Donato, Dipole anisotropy in cosmic electrons and positrons: inspection on local sources, JCAP 1701 (2017) 006, [1611.06237].

[7] T. Delahaye, J. Lavalle, R. Lineros, F. Donato and N. Fornengo, Galactic electrons and positrons at the Earth: new estimate of the primary and secondary fluxes, A\&A $\mathbf{5 2 4}$ (Dec., 2010), [1002.1910].

[8] R. Kappl, A. Reinert and M. W. Winkler, AMS-02 Antiprotons Reloaded, JCAP 1510 (2015) 034.

[9] Y. Genolini, A. Putze, P. Salati and P. D. Serpico, Theoretical uncertainties in extracting cosmic-ray diffusion parameters: the boron-to-carbon ratio, Astron. Astrophys. 580 (2015) A9, [1504. 03134 ].

[10] D. A. Green, Constraints on the distribution of supernova remnants with Galactocentric radius, MNRAS 454 (Dec., 2015) 1517, [1508.02931].

[11] D. Green, A catalogue of 294 Galactic supernova remnants, Bull.Astron.Soc.India 42 (2014) 47.

[12] M. Di Mauro, F. Donato, N. Fornengo and A. Vittino, Dark matter vs. astrophysics in the interpretation of AMS-02 electron and positron data, JCAP 5 (May, 2016) 031, [1507.07001].

[13] H.E.S.S. collaboration, F. Aharonian et al., The energy spectrum of cosmic-ray electrons at TeV energies, Phys. Rev. Lett. 101 (2008) 261104, [0811.3894].

[14] F. A. Aharonian, A. M. Atoyan and H. J. Voelk, High energy electrons and positrons in cosmic rays as an indicator of the existence of a nearby cosmic tevatron, A\&A 294 (Feb., 1995) L41-L44.

[15] H. Alvarez, J. Aparici, J. May and P. Reich, The radio spectral index of the Vela supernova remnant, A\&A 372 (June, 2001) 636-643.

[16] B. Uyanıker, W. Reich, A. Yar and E. Fürst, Radio emission from the Cygnus Loop and its spectral characteristics, A\&A 426 (Nov., 2004) 909-924, [astro-ph / 0409176 ].

[17] H. Katagiri, L. Tibaldo, J. Ballet, F. Giordano, I. A. Grenier, T. A. Porter et al., Fermi Large Area Telescope Observations of the Cygnus Loop Supernova Remnant, ApJ 741 (Nov., 2011) 44.

[18] I. Sushch and B. Hnatyk, Modelling of the radio emission from the Vela supernova remnant, Astron. Astrophys. 561 (2014) A139, [1312.0777]. 